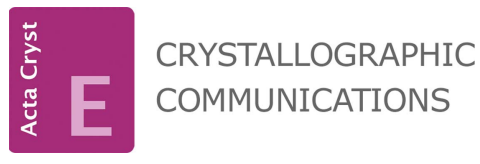

ISSN 2056-9890

Received 24 March 2016

Accepted 25 March 2016

Edited by J. Simpson, University of Otago, New Zealand

Keywords: conjugation; anti-aromatic; hydrogen bonds; layered structure; crystal structure.

CCDC reference: 1470620

Supporting information: this article has supporting information at journals.iucr.org/e

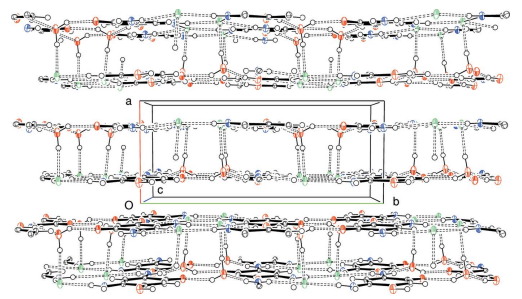

OPEN $\odot$ ACCESS

\section{A purple odyssey: synthesis and structure of 3-amino-4-hydroxy-6-oxocyclohexa-2,4-dien-1- iminium chloride monohydrate}

\author{
M. John Plater and William T. A. Harrison*
}

Department of Chemistry, University of Aberdeen, Aberdeen AB24 3UE, Scotland. *Correspondence e-mail: w.harrison@abdn.ac.uk

In the cation of the title hydrated molecular salt, $\mathrm{C}_{6} \mathrm{H}_{7} \mathrm{~N}_{2} \mathrm{O}_{2}{ }^{+} \cdot \mathrm{Cl}^{-} \cdot \mathrm{H}_{2} \mathrm{O}$, the sixmembered ring shows unequal bond lengths consistent with delocalization of electrons over two separate $6 \pi$ systems with single bonds between them. In the crystal, the components are linked by $\mathrm{N}-\mathrm{H} \cdots \mathrm{Cl}, \mathrm{N}-\mathrm{H} \cdots \mathrm{O}, \mathrm{O}-\mathrm{H} \cdots \mathrm{Cl}$ and $\mathrm{O}-\mathrm{H} \cdots \mathrm{O}$ hydrogen bonds, generating double layers propagating in (100).

\section{Chemical context}

In the course of our ongoing studies (Plater \& Harrison, 2013, 2014a,b; Plater \& Jackson, 2014) on new conjugated products obtained from the oxidation of aromatic amines, we attempted the oxidation of 1,2,4,5-tetraaminobenzene, 1. As long ago as 1887, it was demonstrated (Nietzki \& Hagenbach, 1887) that this compound undergoes aerial oxidation to form 2,5-diamino-1,4-benzoquinonediimine, 2. More recently, Braunstein et al. (2003) have studied the oxidation of compound $\mathbf{1}$ and the related compound 2,4-diaminoresorcinol, $\mathbf{3}$, to synthesize (1E)-N-(2,2-dimethylpropyl)-5-[(2,2-dimethylpropyl)amino]2-hydroxy-4-oxocyclohexa-2,5-dien-1-iminium chloride, 4, which generates the zwitterion $\mathbf{5}$ when treated with base.<smiles>Nc1cc(N)c(N)cc1N</smiles>

1<smiles>CC(C)(C)CNC1=CC(=[NH+]CC(C)(C)C)C(=O)C=C1O</smiles><smiles>N=C1C=C(N)C(=N)C=C1N</smiles>

$$
\stackrel{\text { base }}{\rightleftharpoons \text { acid }}
$$<smiles>Nc1cc(N)c(O)cc1O</smiles><smiles>CC(C)(C)CNC1=CC(=[NH+]CC(C)(C)C)C(=O)C=C1[O-]</smiles>

By careful oxidation of the tetrahydrochloride salt of amine 1 with potassium dichromate, we isolated and crystallized the chloride salt of the parent 3-amino-4-hydroxy-6-oxocyclohexa-2,4-dien-1-iminium cation, $\mathbf{8}$, as a monohydrate $\left[\mathrm{C}_{6} \mathrm{H}_{7} \mathrm{~N}_{2} \mathrm{O}_{2}{ }^{+} \cdot \mathrm{Cl}^{-} \cdot \mathrm{H}_{2} \mathrm{O},(\mathrm{I})\right]$ in the form of purple needles. This reaction must proceed via the elusive intermediate $\mathbf{6}$ which spontaneously hydrolyses. The first hydrolysis product should be intermediate 7. This contains a conjugated iminium salt and a vinylogous amide, which must hydrolyse rapidly, possibly because of the stability of the acidic enol formed. It appears to be a rapid hydrolysis for an amide under mild conditions and so stabilization of a tetrahedral intermediate by the positive iminium salt might occur. 


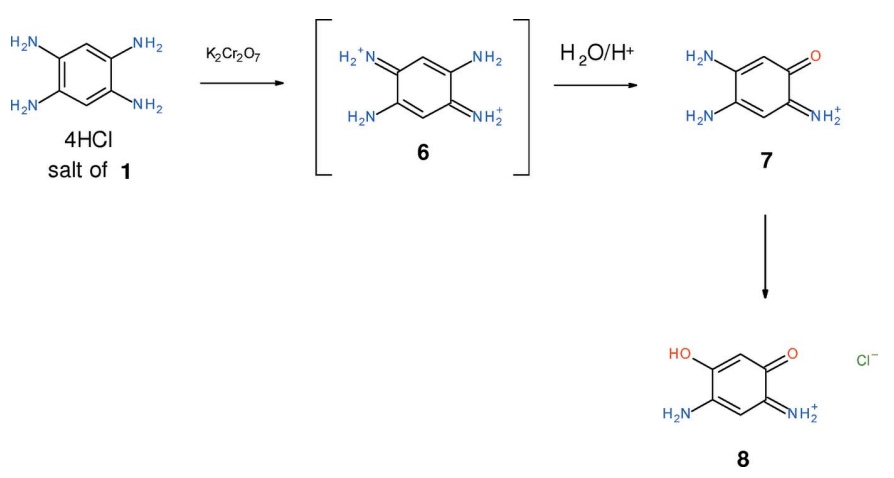

\section{Structural commentary}

The asymmetric unit of (I) consists of one essentially planar $\mathrm{C}_{6} \mathrm{H}_{7} \mathrm{~N}_{2} \mathrm{O}_{2}{ }^{+}$cation (r.m.s. deviation for the non-hydrogen atoms $=0.028 \AA$ ), a chloride counter-ion and a water molecule of crystallization (Fig. 1). Despite being a nominal $6 \pi$ aromatic system, the bond lengths of the C1-C6 ring in (I) are far from equal and are split into three groups of two: the shortest are $\mathrm{C} 1-\mathrm{C} 6$ [1.354 (5)] and $\mathrm{C} 3-\mathrm{C} 4$ [1.381 (5)], followed by C4C5 [1.406 (5)] and C1-C2 [1.436 (5) $⿱$ A]. Finally, the C2-C3 [1.532 (4)] and C5-C6 [1.500(5) $\AA]$ lengths are those expected for a $\mathrm{C}-\mathrm{C} \sigma$ bond.<smiles>NC1=CC(=[NH2+])C(=O)C=C1O</smiles>

(I)

The short $\mathrm{C} 3-\mathrm{C} 4$ and $\mathrm{C} 4-\mathrm{C} 5$ bonds correlate with the approximately equal $\mathrm{C} 3-\mathrm{N} 1 \quad[1.320(4)]$ and $\mathrm{C} 5-\mathrm{N} 2$ [1.306 (4) $\AA$ ] bond lengths, which imply equal delocalization of the positive charge of the cation over atoms N1 and N2, mediated via the $\mathrm{C}-\mathrm{N}$ and $\mathrm{C}-\mathrm{C}$ bonds between them. In terms of the 'oxygen side' of the cation, the $\mathrm{C} 6-\mathrm{O} 2$ bond $[1.320(4) \AA]$ is short for a $\mathrm{C}-\mathrm{O}$ single bond whereas $\mathrm{C} 2-\mathrm{O} 1$

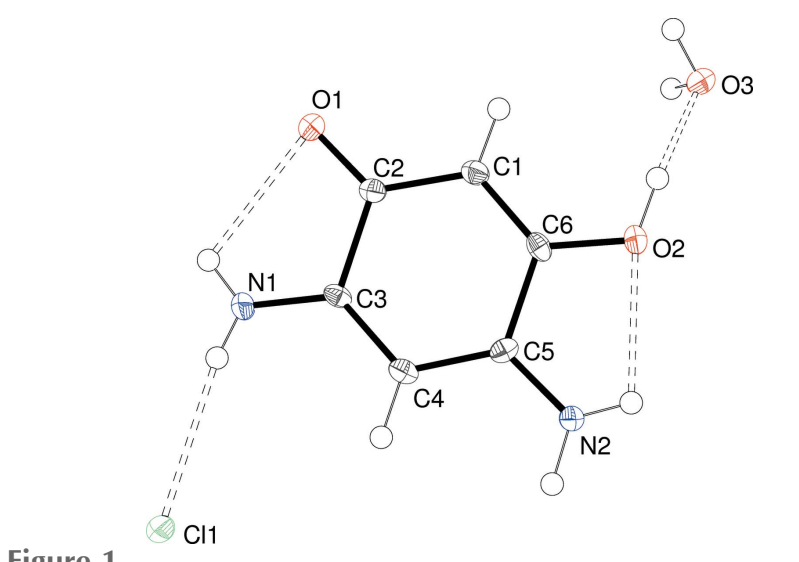

Figure 1

The molecular structure of (I) showing $50 \%$ displacement ellipsoids. Hydrogen bonds are shown as double-dashed lines.
Table 1

Hydrogen-bond geometry $\left(\AA{ }^{\circ}\right)$.

\begin{tabular}{|c|c|c|c|c|}
\hline$D-\mathrm{H} \cdots A$ & $D-\mathrm{H}$ & $\mathrm{H} \cdots A$ & $D \cdots A$ & $D-\mathrm{H} \cdots A$ \\
\hline $\mathrm{N} 1-\mathrm{H} 2 n \cdots \mathrm{O} 1$ & $0.81(5)$ & $2.33(5)$ & $2.653(4)$ & $105(4)$ \\
\hline $\mathrm{N} 2-\mathrm{H} 4 n \cdots \mathrm{O} 2$ & $0.84(4)$ & $2.23(5)$ & $2.595(4)$ & $107(4)$ \\
\hline $\mathrm{N} 1-\mathrm{H} 1 n \cdots \mathrm{Cl} 1$ & $0.80(5)$ & $2.45(5)$ & $3.238(3)$ & $169(4)$ \\
\hline $\mathrm{N} 1-\mathrm{H} 2 n \cdots \mathrm{O}^{\mathrm{i}}$ & $0.81(5)$ & $2.25(5)$ & $3.011(4)$ & $156(4)$ \\
\hline $\mathrm{N} 2-\mathrm{H} 3 n \cdots \mathrm{Cl} 1^{\mathrm{ii}}$ & $0.93(4)$ & $2.22(4)$ & 3.149 (3) & $177(4)$ \\
\hline $\mathrm{N} 2-\mathrm{H} 4 n \cdots \mathrm{Cl} 1^{\mathrm{iii}}$ & $0.83(5)$ & $2.44(5)$ & $3.231(3)$ & $158(4)$ \\
\hline $\mathrm{O} 2-\mathrm{H} 1 o \cdots \mathrm{O} 3$ & $0.92(5)$ & $1.65(5)$ & $2.548(4)$ & $165(4)$ \\
\hline $\mathrm{O} 3-\mathrm{H} 1 w \cdots \mathrm{O} 1^{\mathrm{iv}}$ & $0.88(5)$ & $1.98(5)$ & $2.801(4)$ & $154(4)$ \\
\hline $\mathrm{O} 3-\mathrm{H} 2 w \cdots \mathrm{Cl} 1^{\mathrm{v}}$ & $1.00(5)$ & $2.11(5)$ & $3.116(3)$ & $176(4)$ \\
\hline
\end{tabular}

Symmetry codes: (i) $x, y, z+1$; (ii) $x,-y+\frac{3}{2}, z-\frac{1}{2}$; (iii) $x, y, z-1$; (iv) $x,-y+\frac{1}{2}, z-\frac{1}{2}$; (v) $-x+1,-y+1,-z+1$.

[1.227 (4) $\AA$ ] is slightly lengthened for a nominal $\mathrm{C}=\mathrm{O}$ double bond. This in combination with the $\mathrm{C} 1-\mathrm{C} 2$ and $\mathrm{C} 1-\mathrm{C} 6$ bond lengths again implies a degree of delocalization over these five atoms. However, the long $\mathrm{C} 2-\mathrm{C} 3$ and $\mathrm{C} 5-\mathrm{C} 6$ bonds imply little, if any, conjugation between the two delocalized components $(\mathrm{O} 2 / \mathrm{C} 6 / \mathrm{C} 1 / \mathrm{C} 2 / \mathrm{O} 1$ and $\mathrm{N} 2 / \mathrm{C} 5 / \mathrm{C} 4 / \mathrm{C} 3 / \mathrm{N} 1)$ of the cation.

The cation features two intramolecular $\mathrm{N}-\mathrm{H} \cdots \mathrm{O}$ hydrogen bonds, viz. $\mathrm{N} 1-\mathrm{H} 2 n \cdots \mathrm{O} 1$ and $\mathrm{N} 2-\mathrm{H} 4 n \cdots \mathrm{O} 2$ (Table 1 ), which both close $S(5)$ rings.

\section{Supramolecular features}

In the crystal, the components are linked by $\mathrm{N}-\mathrm{H} \cdots \mathrm{Cl}, \mathrm{N}-$ $\mathrm{H} \cdots \mathrm{O}, \mathrm{O}-\mathrm{H} \cdots \mathrm{Cl}$ and $\mathrm{O}-\mathrm{H} \cdots \mathrm{O}$ hydrogen bonds (Table 1 ). If the cation and chloride anion are considered together, then [001] chains arise (Fig. 2) in which adjacent cations are related to each other by $c$-glide symmetry. Each link in the chain comprises two cations and two anions and $R_{4}^{2}(12)$ loops are apparent.

When the cation and water molecule are considered together, an [001] chain also arises (Fig. 3). The water molecule plays a key role in terms of both accepting hydrogen bonds from $\mathrm{O} 2$ and $\mathrm{N} 1$ and donating a hydrogen bond to $\mathrm{O} 1$ (it also acts as a donor to the chloride ion). The end result is a chain

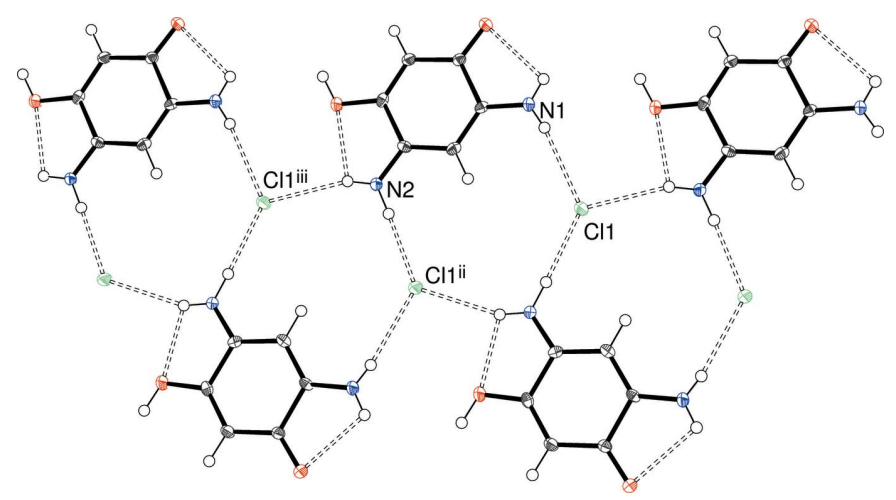

Figure 2

Detail of the crystal structure of (I) showing the formation of [001] chains of cations and chloride ions linked by $\mathrm{N}-\mathrm{H} \cdots \mathrm{Cl}$ hydrogen bonds. Symmetry codes as in Table 1. 


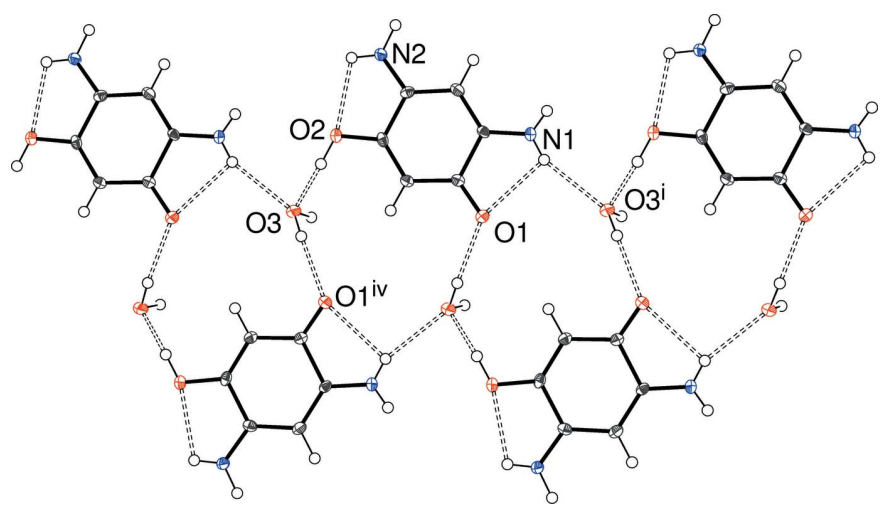

Figure 3

Detail of the crystal structure of (I) showing the formation of [001] chains of cations and water molecules linked by $\mathrm{O}-\mathrm{H} \cdots \mathrm{O}$ and $\mathrm{N}-\mathrm{H} \cdots \mathrm{O}$ hydrogen bonds. Symmetry codes as in Table 1 .

featuring $R_{4}^{4}(12)$ loops (counted via the intramolecular N1$\mathrm{H} 2 n$... O1 hydrogen bond).

When all components are considered together, (100) double sheets result (Fig. 4), with the water-O3-H2w...Cl1 hydrogen bond providing the key link between the sheets. Overall, the chloride ion accepts four hydrogen bonds (three $\mathrm{N}-\mathrm{H} \cdots \mathrm{Cl}$ and one $\mathrm{O}-\mathrm{H} \cdots \mathrm{Cl}$ interactions) in an irregular geometry.

\section{Database survey}

The compound (1E)-N-(2,2-dimethylpropyl)-5-[(2,2-dimethylpropyl)amino]-2-hydroxy-4-oxocyclohexa-2,5-dien-1-iminium chloride chloroform monosolvate (CCDC refcode:
VASVER; Braunstein et al., 2003) was noted in the chemical context section above: these authors discuss its electronic structure in detail including its potentially anti-aromatic character. The crystal structure of the parent unprotonated zwitterion 3-oxo-4-amino-6-iminiophenolate monohydrate (HAZQUV; Yang et al., 2005) is known as are those of a number of its alkylated/functionalized derivatives (Braunstein et al., 2009; Tamboura et al., 2009; Kauf \& Braunstein, 2011) and metal complexes (Paretzki et al., 2010). The carboncarbon bond lengths in the six-membered ring in all these compounds are similar to those seen in (I).

\section{Synthesis and crystallization}

1,2,4,5-Benzenetetraamine tetrahydrochloride (200 mg, $0.7 \mathrm{mmol}$ ) in distilled water $(75 \mathrm{ml})$ was treated with an excess of $\mathrm{K}_{2} \mathrm{Cr}_{2} \mathrm{O}_{7}(140 \mathrm{mg}, 0.48 \mathrm{mmol}, 0.6 \mathrm{eq})$ and stirred at room temperature for $24 \mathrm{~h}$. The brown mixture was neutralized with $\mathrm{NaHCO}_{3}$ giving a brown or red precipitate, which was then extracted with $\mathrm{CH}_{2} \mathrm{Cl}_{2}(10 \times 50 \mathrm{ml})$. The yellow extracts were combined, decanted, then stirred with methanol $(50 \mathrm{ml})$ containing five drops of conc. $\mathrm{HCl}(\mathrm{aq})$. The yellow solution turned purple. This was evaporated to dryness, then the product was dissolved in methanol $(50 \mathrm{ml})$ to yield a red solution and recrystallized by slow evaporation to leave the title compound $(15 \mathrm{mg}, 8 \%)$ as purple needles: m.p. $>473 \mathrm{~K}$; $\lambda_{\max }$ (ethanol)/nm $503(\log \varepsilon 2.90)$ and 325(3.99); $v$ (diamond anvil)/cm ${ }^{-1}$ 2953br, 1688s, 1547vs, 1401vs, 1310vs, 1251vs, $1141 v s, 871 v s, 853 s, 711 v s, 654 v s, 579 v s, 454 s$ and $420 \mathrm{~s} ; \mathrm{m} / \mathrm{z}$ (orbitrap ASAP) $139.0498\left(M^{+}, 100 \%\right), \mathrm{C}_{6} \mathrm{H}_{7} \mathrm{~N}_{2} \mathrm{O}_{2}$ requires 139.0502. The UV/visible spectrum of (I) is shown in Fig. 5.
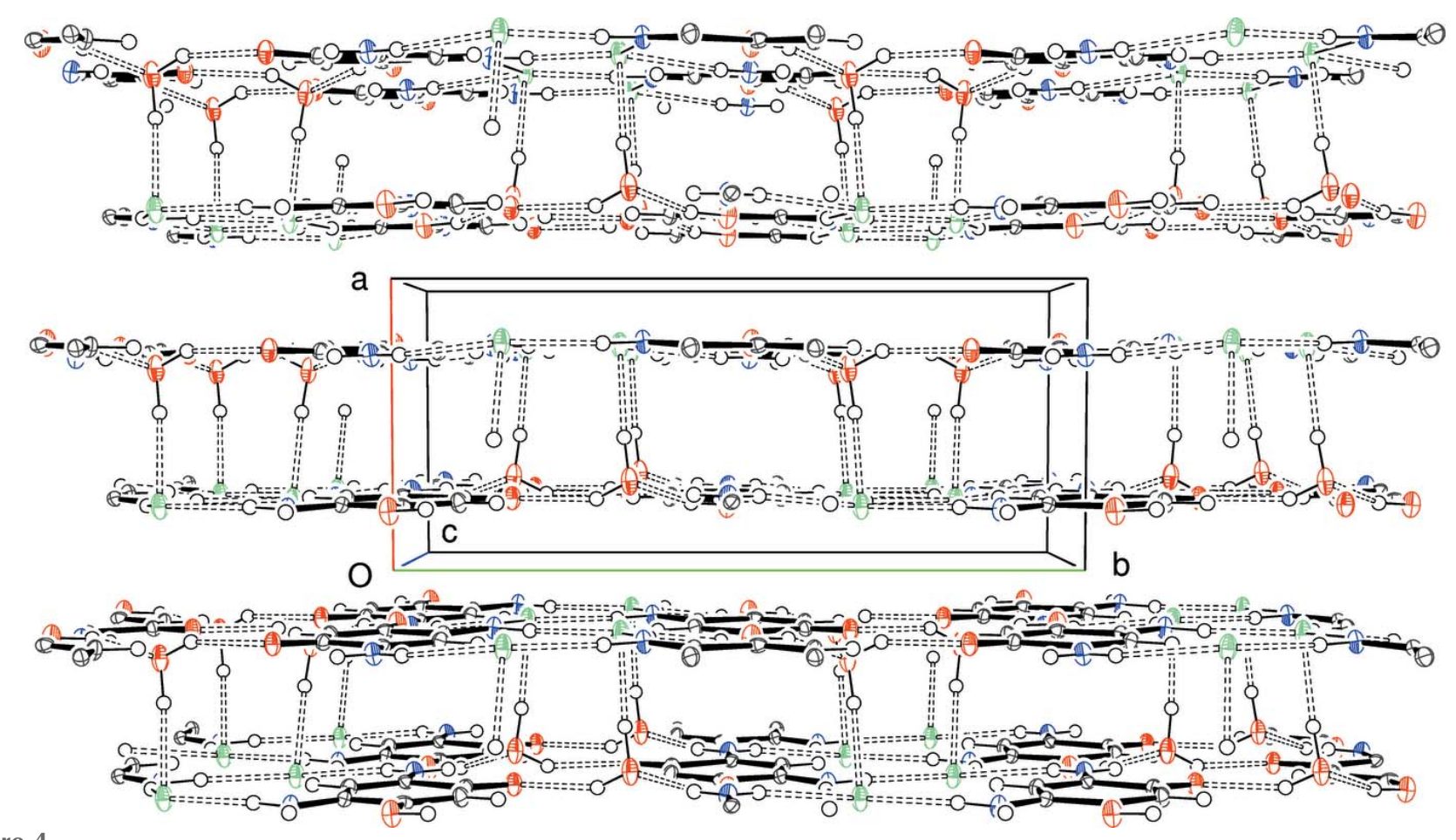

Figure 4

The packing in (I) viewed along [001] showing the formation of (100) double layers. 


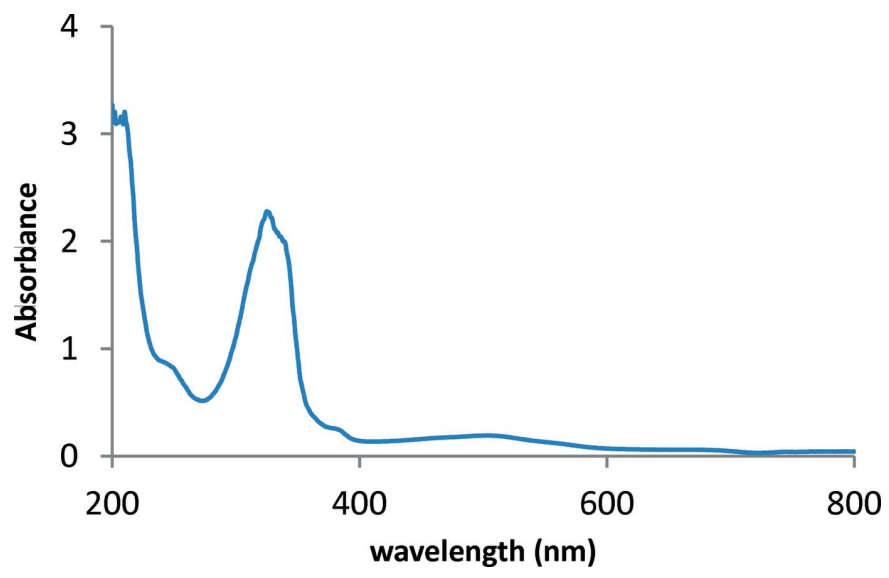

Figure 5

UV/visible spectrum of (I) $\left(2.3 \times 10^{-4} M\right.$ solution in ethanol $)$.

\section{Refinement}

Crystal data, data collection and structure refinement details are summarized in Table 2. The $\mathrm{C}$-bound $\mathrm{H}$ atoms were geometrically placed $(\mathrm{C}-\mathrm{H}=0.95 \AA)$ and refined as riding atoms. The $\mathrm{N}$ - and $\mathrm{O}$-bound $\mathrm{H}$ atoms were located in difference maps and their positions were freely refined. The constraint $U_{\text {iso }}(\mathrm{H})=1.2 U_{\text {eq }}$ (carrier) was applied in all cases. The crystal studied was found to be a twin with the components related by a $180^{\circ}$ rotation about [001].

\section{Acknowledgements}

We thank the EPSRC National Crystallography Service (University of Southampton) for the data collection and the EPSRC National Mass Spectrometry Service (University of Swansea) for the HRMS data.

\section{References}

Braunstein, P., Bubrin, D. \& Sarkar, B. (2009). Inorg. Chem. 48, 2534 2540.

Braunstein, P., Siri, O., Taquet, J.-P., Rohmer, M.-M., Bénard, M. \& Welter, R. (2003). J. Am. Chem. Soc. 125, 12246-12256.

Farrugia, L. J. (2012). J. Appl. Cryst. 45, 849-854.

Kauf, T. \& Braunstein, P. (2011). Inorg. Chem. 50, 11472-11480.

Nietzki, R. \& Hagenbach, E. (1887). Chem. Ber. 20, 328-338.
Table 2

Experimental details.

\begin{tabular}{|c|c|}
\hline \multicolumn{2}{|l|}{ Crystal data } \\
\hline Chemical formula & $\mathrm{C}_{6} \mathrm{H}_{7} \mathrm{~N}_{2} \mathrm{O}_{2} \cdot \mathrm{Cl} \cdot \mathrm{H}_{2} \mathrm{O}$ \\
\hline$M_{\mathrm{r}}$ & 192.60 \\
\hline Crystal system, space group & Monoclinic, $P 2_{1} / c$ \\
\hline Temperature $(\mathrm{K})$ & 100 \\
\hline$a, b, c(\AA)$ & $\begin{array}{l}6.3070(7), 14.9614(18) \\
\quad 8.9198(11)\end{array}$ \\
\hline$\beta\left(^{\circ}\right)$ & $93.457(1)$ \\
\hline$V\left(\AA^{3}\right)$ & $840.15(17)$ \\
\hline$Z$ & 4 \\
\hline Radiation type & Mo $K \alpha$ \\
\hline$\mu\left(\mathrm{mm}^{-1}\right)$ & 0.42 \\
\hline Crystal size $(\mathrm{mm})$ & $0.11 \times 0.04 \times 0.03$ \\
\hline \multicolumn{2}{|l|}{ Data collection } \\
\hline Diffractometer & Rigaku Mercury CCD \\
\hline Absorption correction & - \\
\hline $\begin{array}{l}\text { No. of measured, independent and } \\
\text { observed }[I>2 \sigma(I)] \text { reflections }\end{array}$ & $3102,3102,2789$ \\
\hline $\begin{array}{l}R_{\text {int }} \\
(\sin \theta / \lambda)_{\max }\left(\AA^{-1}\right)\end{array}$ & $\begin{array}{l}? \\
0.651\end{array}$ \\
\hline \multicolumn{2}{|l|}{ Refinement } \\
\hline$R\left[F^{2}>2 \sigma\left(F^{2}\right)\right], w R\left(F^{2}\right), S$ & $0.072,0.159,1.22$ \\
\hline No. of reflections & 3102 \\
\hline No. of parameters & 131 \\
\hline $\mathrm{H}$-atom treatment & $\begin{array}{l}\mathrm{H} \text { atoms treated by a mixture of } \\
\text { independent and constrained } \\
\text { refinement }\end{array}$ \\
\hline$\Delta \rho_{\max }, \Delta \rho_{\min }\left(\mathrm{e} \AA^{-3}\right)$ & $0.57,-0.40$ \\
\hline
\end{tabular}

Computer programs: CrysAlis PRO (Rigaku, 2015), SHELXS97 and SHELXL97 (Sheldrick, 2008), ORTEP-3 (Farrugia, 2012) and publCIF (Westrip, 2010).

Paretzki, A., Pattacini, R., Huebner, R., Braunstein, P. \& Sarkar, B. (2010). Chem. Commun. 46, 1497-1499.

Plater, M. J. \& Harrison, W. T. A. (2013). J. Chem. Res. (S), 37, 427434.

Plater, M. J. \& Harrison, W. T. A. (2014a). J. Chem. Res. (S), 38, 351355.

Plater, M. J. \& Harrison, W. T. A. (2014b). J. Chem. Res. (S), 38, 651654.

Plater, M. J. \& Jackson, T. (2014). J. Chem. Res. (S), 38, 437-442.

Rigaku (2015). CrysAlis PRO. Rigaku Corporation, Tokyo, Japan.

Sheldrick, G. M. (2008). Acta Cryst. A64, 112-122.

Tamboura, F. B., Cazin, C. S. J., Pattacini, R. \& Braunstein, P. (2009). Eur. J. Org. Chem. pp. 3340-3350.

Westrip, S. P. (2010). J. Appl. Cryst. 43, 920-925.

Yang, Q.-Z., Siri, O. \& Braunstein, P. (2005). Chem. Eur. J. 11, $7237-$ 7246. 


\section{supporting information}

Acta Cryst. (2016). E72, 604-607 [doi:10.1107/S2056989016005107]

\section{A purple odyssey: synthesis and structure of 3-amino-4-hydroxy-6-oxocyclo-} hexa-2,4-dien-1-iminium chloride monohydrate

\section{John Plater and William T. A. Harrison}

Computing details

Data collection: CrysAlis PRO (Rigaku, 2015); cell refinement: CrysAlis PRO (Rigaku, 2015); data reduction: CrysAlis PRO (Rigaku, 2015); program(s) used to solve structure: SHELXS97 (Sheldrick, 2008); program(s) used to refine structure: SHELXL97 (Sheldrick, 2008); molecular graphics: ORTEP-3 (Farrugia, 2012); software used to prepare material for publication: publCIF (Westrip, 2010).

3-Amino-4-hydroxy-6-oxocyclohexa-2,4-dien-1-iminium chloride monohydrate

\section{Crystal data}

$\mathrm{C}_{6} \mathrm{H}_{7} \mathrm{~N}_{2} \mathrm{O}_{2} \cdot \mathrm{Cl} \cdot \mathrm{H}_{2} \mathrm{O}$

$M_{r}=192.60$

Monoclinic, $P 2_{1} / c$

$a=6.3070$ (7) $\AA$

$b=14.9614$ (18) $\AA$

$c=8.9198(11) \AA$

$\beta=93.457(1)^{\circ}$

$V=840.15(17) \AA^{3}$

$Z=4$

Data collection

Rigaku Mercury CCD

diffractometer

$\omega$ scans

3102 measured reflections

3102 independent reflections

Refinement

Refinement on $F^{2}$

Least-squares matrix: full

$R\left[F^{2}>2 \sigma\left(F^{2}\right)\right]=0.072$

$w R\left(F^{2}\right)=0.159$

$S=1.22$

3102 reflections

131 parameters

0 restraints
$F(000)=400$

$D_{\mathrm{x}}=1.523 \mathrm{Mg} \mathrm{m}^{-3}$

Mo $K \alpha$ radiation, $\lambda=0.71073 \AA$

Cell parameters from 1747 reflections

$\theta=3.2-27.5^{\circ}$

$\mu=0.42 \mathrm{~mm}^{-1}$

$T=100 \mathrm{~K}$

Rod, purple

$0.11 \times 0.04 \times 0.03 \mathrm{~mm}$

2789 reflections with $I>2 \sigma(I)$

$\theta_{\text {max }}=27.6^{\circ}, \theta_{\min }=2.7^{\circ}$

$h=-8 \rightarrow 8$

$k=-19 \rightarrow 19$

$l=-7 \rightarrow 11$

Primary atom site location: structure-invariant direct methods

Hydrogen site location: difference Fourier map

$\mathrm{H}$ atoms treated by a mixture of independent and constrained refinement

$w=1 /\left[\sigma^{2}\left(F_{\mathrm{o}}^{2}\right)+(0.0472 P)^{2}+1.7994 P\right]$ where $P=\left(F_{\mathrm{o}}^{2}+2 F_{\mathrm{c}}^{2}\right) / 3$

$(\Delta / \sigma)_{\max }<0.001$

$\Delta \rho_{\max }=0.56 \mathrm{e} \AA^{-3}$

$\Delta \rho_{\min }=-0.39$ e $\AA^{-3}$ 


\section{Special details}

Geometry. All esds (except the esd in the dihedral angle between two 1.s. planes) are estimated using the full covariance matrix. The cell esds are taken into account individually in the estimation of esds in distances, angles and torsion angles; correlations between esds in cell parameters are only used when they are defined by crystal symmetry. An approximate (isotropic) treatment of cell esds is used for estimating esds involving l.s. planes.

Refinement. Refined as a 2 -component twin $\left(180^{\circ}\right.$ rotation about [001])

Fractional atomic coordinates and isotropic or equivalent isotropic displacement parameters $\left(\hat{A}^{2}\right)$

\begin{tabular}{lllll}
\hline & $x$ & $y$ & $z$ & $U_{\text {iso }} / U_{\mathrm{eq}}$ \\
\hline $\mathrm{C} 1$ & $0.2403(6)$ & $0.3972(2)$ & $0.3386(4)$ & $0.0152(7)$ \\
$\mathrm{H} 1$ & 0.2413 & 0.3419 & 0.2865 & $0.018^{*}$ \\
$\mathrm{C} 2$ & $0.2473(6)$ & $0.3979(2)$ & $0.4998(4)$ & $0.0132(7)$ \\
$\mathrm{C} 3$ & $0.2536(6)$ & $0.4888(2)$ & $0.5792(4)$ & $0.0120(7)$ \\
$\mathrm{C} 4$ & $0.2459(6)$ & $0.5671(2)$ & $0.4970(4)$ & $0.0155(7)$ \\
$\mathrm{H} 4$ & 0.2493 & 0.6233 & 0.5468 & $0.019^{*}$ \\
$\mathrm{C} 5$ & $0.2332(6)$ & $0.5632(2)$ & $0.3392(4)$ & $0.0133(7)$ \\
$\mathrm{C} 6$ & $0.2324(6)$ & $0.4747(2)$ & $0.2601(4)$ & $0.0133(7)$ \\
N1 & $0.2648(6)$ & $0.4838(2)$ & $0.7271(3)$ & $0.0165(7)$ \\
H1n & $0.265(7)$ & $0.527(3)$ & $0.779(5)$ & $0.020^{*}$ \\
H2n & $0.270(7)$ & $0.436(3)$ & $0.770(5)$ & $0.020^{*}$ \\
N2 & $0.2212(6)$ & $0.6344(2)$ & $0.2543(4)$ & $0.0162(7)$ \\
H3n & $0.227(7)$ & $0.690(3)$ & $0.300(5)$ & $0.019^{*}$ \\
H4n & $0.216(7)$ & $0.629(3)$ & $0.161(5)$ & $0.019^{*}$ \\
O1 & $0.2459(4)$ & $0.33037(17)$ & $0.5778(3)$ & $0.0184(6)$ \\
O2 & $0.2219(5)$ & $0.48297(18)$ & $0.1125(3)$ & $0.0201(6)$ \\
H1o & $0.230(7)$ & $0.430(3)$ & $0.063(5)$ & $0.024^{*}$ \\
C11 & $0.22638(16)$ & $0.67344(6)$ & $0.89828(10)$ & $0.0208(3)$ \\
O3 & $0.3077(5)$ & $0.34226(17)$ & $-0.0329(3)$ & $0.0223(6)$ \\
H1w & $0.247(8)$ & $0.294(3)$ & $-0.001(5)$ & $0.027^{*}$ \\
H2w & $0.458(8)$ & $0.334(3)$ & $0.008(6)$ & $0.027^{*}$ \\
& & & & \\
\hline
\end{tabular}

Atomic displacement parameters $\left(\AA^{2}\right)$

\begin{tabular}{lllllll}
\hline & $U^{11}$ & $U^{22}$ & $U^{33}$ & $U^{12}$ & $U^{13}$ & $U^{23}$ \\
\hline C1 & $0.019(2)$ & $0.0121(15)$ & $0.0149(17)$ & $0.0016(14)$ & $0.0001(14)$ & $-0.0023(13)$ \\
C2 & $0.0134(18)$ & $0.0119(15)$ & $0.0146(17)$ & $-0.0007(14)$ & $0.0013(14)$ & $-0.0015(13)$ \\
C3 & $0.0101(17)$ & $0.0119(15)$ & $0.0137(16)$ & $0.0012(13)$ & $-0.0030(13)$ & $-0.0036(12)$ \\
C4 & $0.0170(19)$ & $0.0134(16)$ & $0.0161(17)$ & $-0.0001(15)$ & $0.0001(14)$ & $-0.0038(13)$ \\
C5 & $0.0118(18)$ & $0.0113(15)$ & $0.0168(17)$ & $-0.0006(14)$ & $0.0005(14)$ & $0.0021(13)$ \\
C6 & $0.0105(18)$ & $0.0179(16)$ & $0.0112(16)$ & $0.0006(14)$ & $-0.0014(13)$ & $-0.0031(13)$ \\
N1 & $0.0243(19)$ & $0.0150(14)$ & $0.0101(15)$ & $0.0015(14)$ & $-0.0005(13)$ & $-0.0001(12)$ \\
N2 & $0.0246(19)$ & $0.0122(13)$ & $0.0120(15)$ & $-0.0015(13)$ & $0.0038(13)$ & $-0.0003(12)$ \\
O1 & $0.0271(15)$ & $0.0137(11)$ & $0.0143(12)$ & $0.0001(12)$ & $0.0017(11)$ & $0.0015(10)$ \\
O2 & $0.0335(18)$ & $0.0165(13)$ & $0.0103(12)$ & $0.0016(12)$ & $0.0008(11)$ & $-0.0001(10)$ \\
C11 & $0.0343(5)$ & $0.0136(4)$ & $0.0144(4)$ & $-0.0010(4)$ & $-0.0010(4)$ & $0.0012(3)$ \\
O3 & $0.0368(18)$ & $0.0133(13)$ & $0.0170(14)$ & $-0.0015(12)$ & $0.0034(12)$ & $0.0015(10)$ \\
& & & & & &
\end{tabular}


Geometric parameters $\left(\AA,{ }^{\circ}\right)$

\begin{tabular}{|c|c|c|c|}
\hline $\mathrm{C} 1-\mathrm{C} 6$ & $1.354(5)$ & $\mathrm{C} 5-\mathrm{C} 6$ & $1.500(5)$ \\
\hline $\mathrm{C} 1-\mathrm{C} 2$ & $1.436(5)$ & $\mathrm{C} 6-\mathrm{O} 2$ & $1.320(4)$ \\
\hline $\mathrm{C} 1-\mathrm{H} 1$ & 0.9500 & $\mathrm{~N} 1-\mathrm{H} 1 \mathrm{n}$ & $0.80(5)$ \\
\hline $\mathrm{C} 2-\mathrm{O} 1$ & $1.227(4)$ & $\mathrm{N} 1-\mathrm{H} 2 \mathrm{n}$ & $0.81(5)$ \\
\hline $\mathrm{C} 2-\mathrm{C} 3$ & $1.532(4)$ & $\mathrm{N} 2-\mathrm{H} 3 \mathrm{n}$ & $0.93(4)$ \\
\hline $\mathrm{C} 3-\mathrm{N} 1$ & $1.320(4)$ & $\mathrm{N} 2-\mathrm{H} 4 \mathrm{n}$ & $0.83(5)$ \\
\hline $\mathrm{C} 3-\mathrm{C} 4$ & $1.381(5)$ & $\mathrm{O} 2-\mathrm{H} 1 \mathrm{o}$ & $0.92(5)$ \\
\hline $\mathrm{C} 4-\mathrm{C} 5$ & $1.406(5)$ & $\mathrm{O} 3-\mathrm{H} 1 \mathrm{w}$ & $0.88(5)$ \\
\hline $\mathrm{C} 4-\mathrm{H} 4$ & 0.9500 & $\mathrm{O} 3-\mathrm{H} 2 \mathrm{w}$ & $1.00(5)$ \\
\hline $\mathrm{C} 5-\mathrm{N} 2$ & $1.306(4)$ & & \\
\hline $\mathrm{C} 6-\mathrm{C} 1-\mathrm{C} 2$ & $120.6(3)$ & $\mathrm{N} 2-\mathrm{C} 5-\mathrm{C} 6$ & $116.6(3)$ \\
\hline $\mathrm{C} 6-\mathrm{C} 1-\mathrm{H} 1$ & 119.7 & $\mathrm{C} 4-\mathrm{C} 5-\mathrm{C} 6$ & $120.4(3)$ \\
\hline $\mathrm{C} 2-\mathrm{C} 1-\mathrm{H} 1$ & 119.7 & $\mathrm{O} 2-\mathrm{C} 6-\mathrm{C} 1$ & $126.4(3)$ \\
\hline $\mathrm{O} 1-\mathrm{C} 2-\mathrm{C} 1$ & $124.1(3)$ & $\mathrm{O} 2-\mathrm{C} 6-\mathrm{C} 5$ & $112.6(3)$ \\
\hline $\mathrm{O} 1-\mathrm{C} 2-\mathrm{C} 3$ & $118.0(3)$ & $\mathrm{C} 1-\mathrm{C} 6-\mathrm{C} 5$ & $120.9(3)$ \\
\hline $\mathrm{C} 1-\mathrm{C} 2-\mathrm{C} 3$ & $117.9(3)$ & $\mathrm{C} 3-\mathrm{N} 1-\mathrm{H} 1 \mathrm{n}$ & $122(3)$ \\
\hline $\mathrm{N} 1-\mathrm{C} 3-\mathrm{C} 4$ & $125.2(3)$ & $\mathrm{C} 3-\mathrm{N} 1-\mathrm{H} 2 \mathrm{n}$ & $121(3)$ \\
\hline $\mathrm{N} 1-\mathrm{C} 3-\mathrm{C} 2$ & $114.2(3)$ & $\mathrm{H} 1 \mathrm{n}-\mathrm{N} 1-\mathrm{H} 2 \mathrm{n}$ & $117(4)$ \\
\hline $\mathrm{C} 4-\mathrm{C} 3-\mathrm{C} 2$ & $120.6(3)$ & $\mathrm{C} 5-\mathrm{N} 2-\mathrm{H} 3 \mathrm{n}$ & $119(3)$ \\
\hline $\mathrm{C} 3-\mathrm{C} 4-\mathrm{C} 5$ & $119.6(3)$ & $\mathrm{C} 5-\mathrm{N} 2-\mathrm{H} 4 \mathrm{n}$ & $120(3)$ \\
\hline $\mathrm{C} 3-\mathrm{C} 4-\mathrm{H} 4$ & 120.2 & $\mathrm{H} 3 \mathrm{n}-\mathrm{N} 2-\mathrm{H} 4 \mathrm{n}$ & $121(4)$ \\
\hline $\mathrm{C} 5-\mathrm{C} 4-\mathrm{H} 4$ & 120.2 & $\mathrm{C} 6-\mathrm{O} 2-\mathrm{H} 1 \mathrm{o}$ & $114(3)$ \\
\hline $\mathrm{N} 2-\mathrm{C} 5-\mathrm{C} 4$ & $123.0(3)$ & $\mathrm{H} 1 \mathrm{w}-\mathrm{O} 3-\mathrm{H} 2 \mathrm{w}$ & $102(4)$ \\
\hline $\mathrm{C} 6-\mathrm{C} 1-\mathrm{C} 2-\mathrm{O} 1$ & $176.9(4)$ & $\mathrm{C} 3-\mathrm{C} 4-\mathrm{C} 5-\mathrm{N} 2$ & $178.6(4)$ \\
\hline $\mathrm{C} 6-\mathrm{C} 1-\mathrm{C} 2-\mathrm{C} 3$ & $-2.2(5)$ & $\mathrm{C} 3-\mathrm{C} 4-\mathrm{C} 5-\mathrm{C} 6$ & $-1.3(6)$ \\
\hline $\mathrm{O} 1-\mathrm{C} 2-\mathrm{C} 3-\mathrm{N} 1$ & $2.4(5)$ & $\mathrm{C} 2-\mathrm{C} 1-\mathrm{C} 6-\mathrm{O} 2$ & $-178.8(4)$ \\
\hline $\mathrm{C} 1-\mathrm{C} 2-\mathrm{C} 3-\mathrm{N} 1$ & $-178.5(3)$ & $\mathrm{C} 2-\mathrm{C} 1-\mathrm{C} 6-\mathrm{C} 5$ & $0.7(6)$ \\
\hline $\mathrm{O} 1-\mathrm{C} 2-\mathrm{C} 3-\mathrm{C} 4$ & $-177.1(4)$ & $\mathrm{N} 2-\mathrm{C} 5-\mathrm{C} 6-\mathrm{O} 2$ & $0.8(5)$ \\
\hline $\mathrm{C} 1-\mathrm{C} 2-\mathrm{C} 3-\mathrm{C} 4$ & $2.0(5)$ & $\mathrm{C} 4-\mathrm{C} 5-\mathrm{C} 6-\mathrm{O} 2$ & $-179.3(3)$ \\
\hline $\mathrm{N} 1-\mathrm{C} 3-\mathrm{C} 4-\mathrm{C} 5$ & $-179.7(4)$ & $\mathrm{N} 2-\mathrm{C} 5-\mathrm{C} 6-\mathrm{C} 1$ & $-178.8(4)$ \\
\hline $\mathrm{C} 2-\mathrm{C} 3-\mathrm{C} 4-\mathrm{C} 5$ & $-0.3(5)$ & $\mathrm{C} 4-\mathrm{C} 5-\mathrm{C} 6-\mathrm{C} 1$ & $1.1(6)$ \\
\hline
\end{tabular}

Hydrogen-bond geometry $\left(A,{ }^{\circ}\right)$

\begin{tabular}{lllll}
\hline$D-\mathrm{H} \cdots A$ & $D-\mathrm{H}$ & $\mathrm{H} \cdots A$ & $D \cdots A$ & $D-\mathrm{H} \cdots A$ \\
\hline $\mathrm{N} 1-\mathrm{H} 2 n \cdots \mathrm{O} 1$ & $0.81(5)$ & $2.33(5)$ & $2.653(4)$ & $105(4)$ \\
$\mathrm{N} 2-\mathrm{H} 4 n \cdots \mathrm{O} 2$ & $0.84(4)$ & $2.23(5)$ & $2.595(4)$ & $107(4)$ \\
$\mathrm{N} 1-\mathrm{H} 1 n \cdots \mathrm{C} 11$ & $0.80(5)$ & $2.45(5)$ & $3.238(3)$ & $169(4)$ \\
$\mathrm{N} 1-\mathrm{H} 2 n \cdots \mathrm{O} 3{ }^{\mathrm{i}}$ & $0.81(5)$ & $2.25(5)$ & $3.011(4)$ & $156(4)$ \\
$\mathrm{N} 2-\mathrm{H} 3 n \cdots \mathrm{C} 11^{1 i}$ & $0.93(4)$ & $2.22(4)$ & $3.149(3)$ & $177(4)$ \\
$\mathrm{N} 2-\mathrm{H} 4 n \cdots \mathrm{C} 11^{i i i}$ & $0.83(5)$ & $2.44(5)$ & $3.231(3)$ & $158(4)$ \\
$\mathrm{O} 2-\mathrm{H} 10 \cdots \mathrm{O} 3$ & $0.92(5)$ & $1.65(5)$ & $2.548(4)$ & $165(4)$
\end{tabular}


supporting information

$\begin{array}{llllr}\mathrm{O} 3-\mathrm{H} 1 w \cdots \mathrm{O} 1^{\text {iv }} & 0.88(5) & 1.98(5) & 2.801(4) & 154(4) \\ \mathrm{O} 3-\mathrm{H} 2 w^{\cdots \cdots} \mathrm{Cl1}^{\mathrm{v}} & 1.00(5) & 2.11(5) & 3.116(3) & 176(4)\end{array}$

Symmetry codes: (i) $x, y, z+1$; (ii) $x,-y+3 / 2, z-1 / 2$; (iii) $x, y, z-1$; (iv) $x,-y+1 / 2, z-1 / 2$; (v) $-x+1,-y+1,-z+1$. 